\title{
A Molecular Dynamics Study on Wrinkles in Graphene with Simply Supported Boundary under In-Plane Shear
}

\author{
Jianzhang Huang and Qiang Han \\ Department of Engineering Mechanics, School of Civil Engineering and Transportation, South China University of Technology, \\ Guangzhou, Guangdong Province 510640, China
}

Correspondence should be addressed to Qiang Han; emqhan@scut.edu.cn

Received 11 November 2016; Revised 17 January 2017; Accepted 7 February 2017; Published 7 March 2017

Academic Editor: Susheng Tan

Copyright (C) 2017 Jianzhang Huang and Qiang Han. This is an open access article distributed under the Creative Commons Attribution License, which permits unrestricted use, distribution, and reproduction in any medium, provided the original work is properly cited.

\begin{abstract}
The formation and evolution mechanisms of wrinkling in a rectangular single layer graphene sheet (SLGS) with simply supported boundary subjected to in-plane shear displacements are investigated using molecular dynamics simulations. Through investigating the out-of-plane displacements of the key point atom, we clarify the wrinkling growth and propagation process. Our results show that the boundary condition plays important roles in the wrinkling deformation. And the dependence of wrinkling parameters on the applied shear displacements is captured. Based on the elasticity theory, the formation mechanism of graphene wrinkling is revealed from the viewpoint of elastic energy. The effects of aspect ratio of graphene, temperature, and loading velocity on graphene wrinkling parameters and patterns are also investigated.
\end{abstract}

\section{Introduction}

Graphene, a research hotspot of nanoengineering, is an important constituent part of nanoelectromechanical systems due to its remarkable mechanical, thermal, and electrical properties [1]. Its fantastic physical properties make graphene an ideal candidate component for nanodevices, such as field-effect transistor, nanosensors, and supercapacitor [25]. As the real 2-dimensional (2D) material with atomic thickness, graphene is found to wrinkle easily because of its relatively low bending rigidity $[6,7]$. Wrinkles can be induced on graphene by several reasons such as cooling-heating treatment, surface morphology growth of substrate, surface structural of substrate, topological defects of graphene, mechanical strain, and the boundary conditions [8-12]. Wrinkling, a kind of structural instability, certainly affects the graphenes remarkable physical property greatly, that is, mechanical, electrical, and thermal properties [13-16]. Dong et al. reported that the wrinkled graphene significantly increased the rate capability and cycling ability of MoO3C-reduced graphene oxide hybrid nanobelts, because the wrinkling morphology of graphene provides continuous electron pathways and stress buffering effects [17]. It is found that the wrinkle size and its top curvature improve the field enhancement factor of the graphene and decrease the electron affinities and ionization potentials [15]. Qin et al. investigated the mechanical properties of wrinkled graphene generated by topological defects and found that the interlayer shear mechanical properties are significantly enhanced due to the geometrical locking effect [18]. Meanwhile, it is possible to control the configuration and morphology of graphene wrinkles to satisfy the demand of nanoengineering applications $[9,19-21]$. Therefore, understanding the deformation mechanism and controlling method of graphene wrinkling have fatal research significance.

The edge boundary condition greatly influences the wrinkling morphology in graphene [10,11]. But to the best of our knowledge, up to now it is not clear of the effects of simply supported boundary condition on graphene wrinkling. To fill the knowledge gap with regard to the simply supported boundary on graphene wrinkling, we investigate the wrinkling mechanism of shear-loaded zigzag rectangular SLGS with simply supported boundary condition investigated based on molecular dynamics (MD) simulations in this paper. The wrinkling deformation mechanism is elaborated through chasing the development of the atomic out-of-plane 


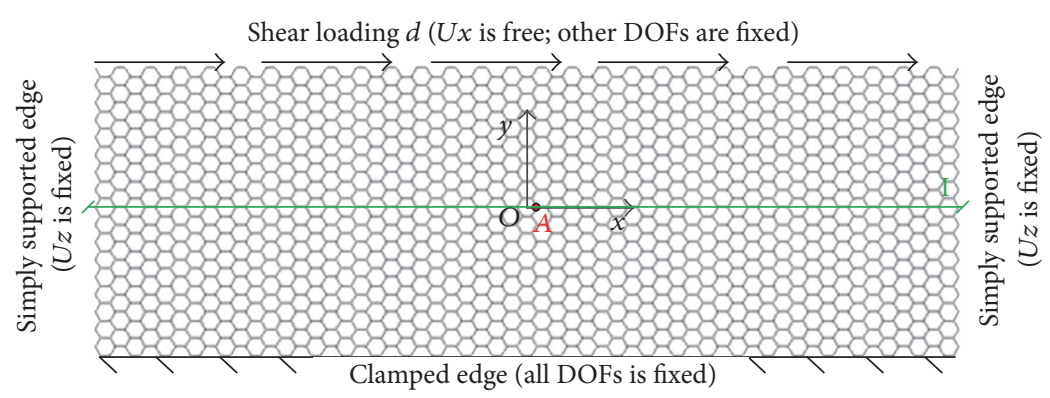

FIGURE 1: Graphene model with given edge boundary condition.

displacement development of the key atom and wrinkling parameters. The formation mechanism of wrinkling is also discussed by elasticity theory. We also investigate the effects of aspect ratio, temperature, and loading velocity on graphene wrinkling in this paper.

\section{Simulation Model and Method}

Figure 1 shows a zigzag rectangular graphene sheet model $\left(12.053 \times 4.052 \mathrm{~nm}^{2}\right)$ with specific edge boundary conditions subjected to in-plane shear. The shear displacement loading is applied on its top layer of atoms, and all the degrees of freedom of the bottom layer atoms are fixed. The left and right edges of graphene are simply supported boundary, of which only the degree of freedom in $z$-direction is fixed. The boundary condition can be expressed by

$$
w=0, \quad \text { if } x=-l \text { or } l
$$

where $w$ is the out-of-plane displacement and the length of graphene is $2 l$. According to the elasticity theory, the wrinkling stability problem of graphene can be represented by a governing equation. The critical wrinkling strains and wrinkling modes can be obtained through the solution of the governing equation. The boundary condition affects the stress distribution in the graphene and has decisive influence on the wrinkling stability and modes. Meanwhile, the boundary condition plays a crucial role in the solution of governing equation of wrinkling problem. Therefore, the wrinkling stability is very sensitive to the boundary condition.

A key point $A(0.1418 \mathrm{~nm} ; 0.0614 \mathrm{~nm})$ and section line I $(y=0.0614 \mathrm{~nm})$ are set upon the graphene surface as seen in Figure 1. The $\mathrm{C}-\mathrm{C}$ bond interaction is described using AIREBO potential [22] and the length of C-C bond is $0.141 \mathrm{~nm}$ with the cut-off distance of $0.2 \mathrm{~nm}$. We employ the MD simulation using the large-scale atomic/molecular massively parallel simulator (LAMMPS) with a time step of $1 \mathrm{fs}$ for integration. The temperature is maintained at $0.01 \mathrm{~K}$ using the Nose-Hoover thermostat. After the equilibration, the shear strain is loaded in the way of applying shear displacements $d$ on the loading edge $0.01 \mathrm{~nm}$ at every 10000 steps and repeated 200 times.

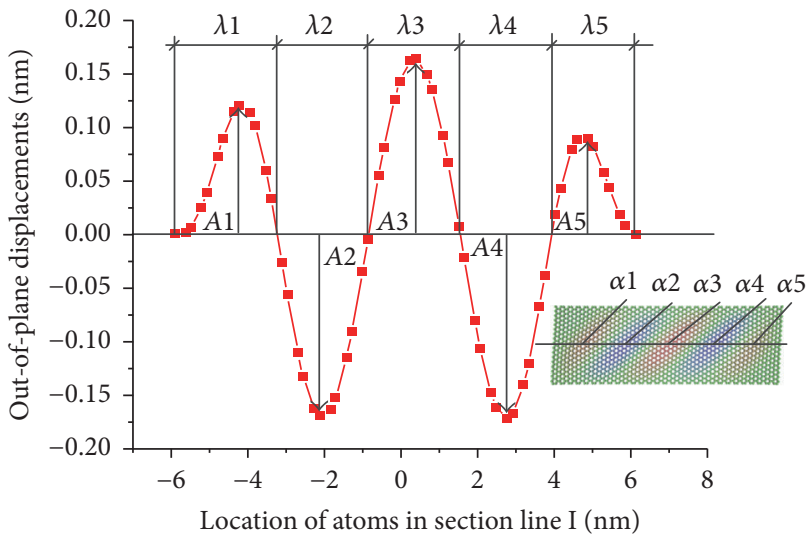

FIGURE 2: Wrinkling parameters of cross-line I.

\section{Results and Discussion}

Self-similar wrinkles with distinct direction angle are generated on the graphene surface by the external mechanical strain. We define the global wrinkling parameters $\overline{\eta_{\chi}}$ to describe the wrinkling characteristics. This $\overline{\eta_{\chi}}$ is used to reflect the mean value of the wrinkling characteristics value defined as the following expression:

$$
\overline{\eta_{\chi}}=\frac{1}{n} \sum_{i=1}^{n} \eta_{\chi i} .
$$

As shown in Figure 2, $\eta_{\chi}$ represents an arbitrary specific wrinkling parameter, such as wrinkling amplitude $\eta_{A}$, wavelength $\eta_{\lambda}$, the ratio of wrinkling amplitude to wavelength $\eta_{A} / \eta_{\lambda}$, and the wrinkling direction angle $\eta_{\alpha}$ as well. The wrinkling direction angle $\eta_{\alpha}$ is defined as the angle between wrinkling direction and $x$-axis direction. The parameter $\eta_{A} / \eta_{\lambda}$ may reflect the morphology of the graphene wrinkles. The wrinkling number $n$ represents the total number of wrinkling crests and troughs. For example, $n=7(4,3)$ indicates that there are 7 wrinkles including 4 wrinkling crests and 3 troughs.

As shown in Figure 3, based on the development of atomic out-of-plane displacement of the key point $A$, the wrinkling developing evolution can be divided into five stages, that is (I) prophase stage, (II) prometaphase stage, (III) metaphase stage, (IV) anaphase stage, and (V) telophase stage. Graphene structure is in a stable stage at first after the 

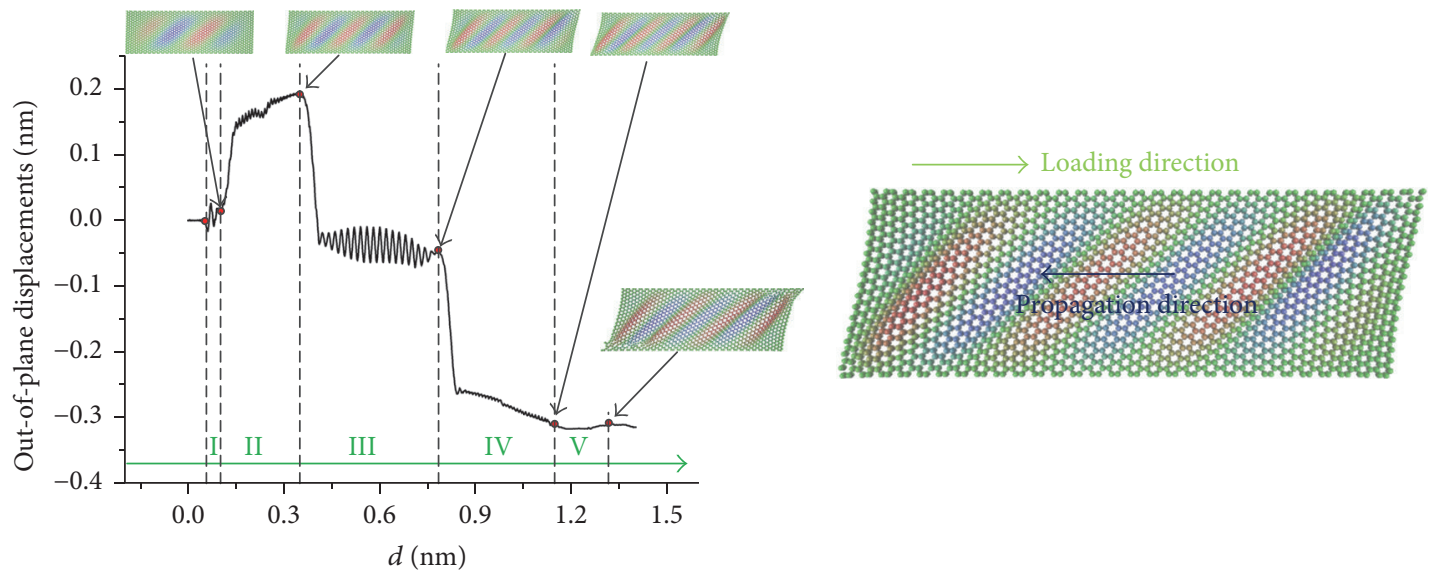

FIGURE 3: Out-of-plane displacements of key point $A$ and wrinkling propagation.

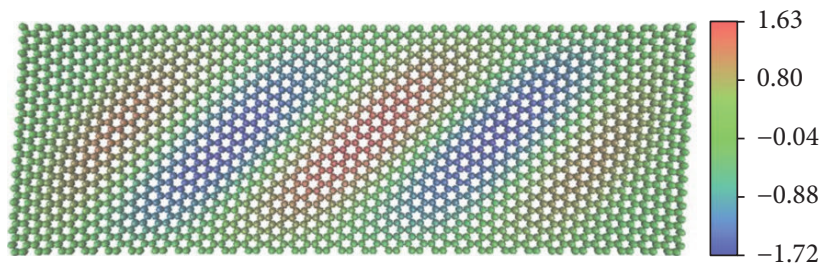

(a) $d=0.21 \mathrm{~nm}$

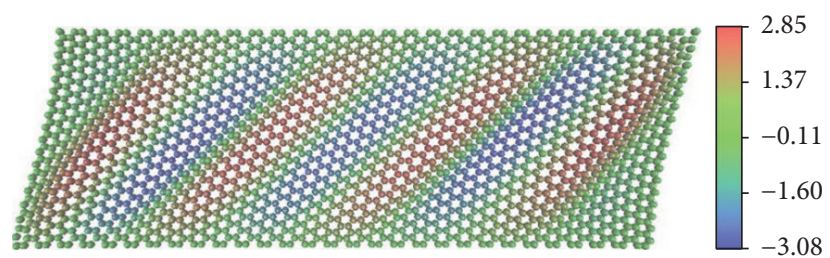

(c) $d=0.88 \mathrm{~nm}$

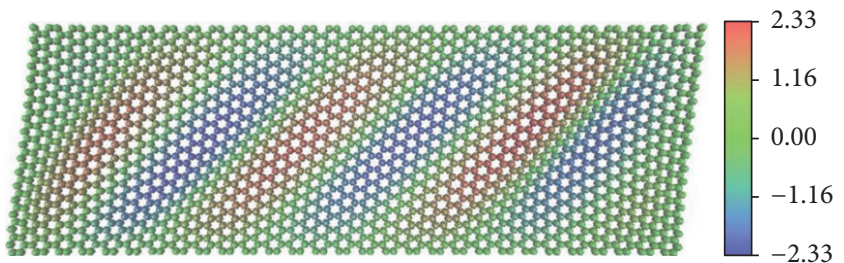

(b) $d=0.44 \mathrm{~nm}$

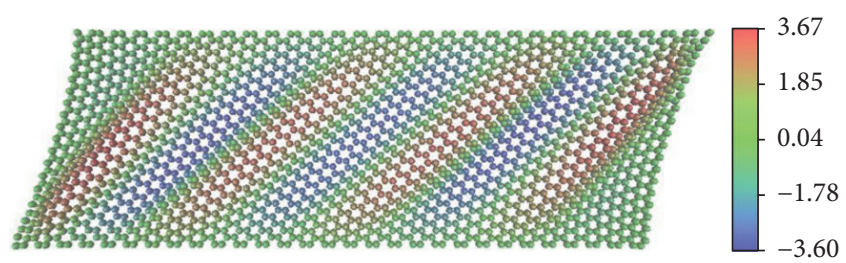

(d) $d=1.24 \mathrm{~nm}$

Figure 4: Wrinkling patterns at different $d$ (unit: $\AA$ ).

shear displacements load. As the $d$ increases to $0.054 \mathrm{~nm}$, wrinkles are formed on the graphene surface and wrinkling evolution begins. Prophase stage occurs in $0.054 \mathrm{~nm} \leq$ $d \leq 0.106 \mathrm{~nm}$, during which graphene wrinkles rapidly and wrinkling numbers are $4(2,2)$ as shown in Figure 3 . When $d$ reaches $0.106 \mathrm{~nm}$, a new wrinkling crest is formed from the right simply supported edge increasing the wrinkling numbers to $5(3,2)$ as shown in Figure $4(\mathrm{a})$ and evolution goes into prometaphase stage, which happens in $0.106 \mathrm{~nm}<$ $d \leq 0.353 \mathrm{~nm}$. After this, the evolution process is pushed into the metaphase stage $(0.353 \mathrm{~nm}<d \leq 0.782 \mathrm{~nm})$. In this stage, a wrinkling trough is generated from the right edge as shown in Figure 4(b) and $n=6(3,3)$. The anaphase stage starts with $d=1.151 \mathrm{~nm}$ and terminates by $d=1.151 \mathrm{~nm}$. During anaphase stage, wrinkling numbers rise to $7(4,3)$ owing to the formation of the new wrinkling crest from the right edge as shown in Figure 4(c). When $d$ reaches $1.151 \mathrm{~nm}$, the evolution process is driven into the last stage, telophase. During the previous four stages, the reciprocating change of key point atomic displacements means that wrinkles develop unstably. Unlike the previous four stages, in telophase stage, wrinkling angle decreases and wrinkling number keeps 7 (4, 3 ) as shown in Figure 4(d) and wrinkling grows stably till the graphene breaks, corresponding to $d=1.316 \mathrm{~nm}$.

Table 1 lists the computing wrinkling parameters results of cross-line I. It is seen that the wrinkling amplitude $\overline{\eta_{A}}$ and ratio of amplitude to wavelength $\overline{\eta_{A} / \eta_{\lambda}}$ grow with the increasing $d$, but the wrinkling wavelength $\overline{\eta_{\lambda}}$ shows a reduction during wrinkling evolution process, indicating that the wrinkles are becoming slender. It is found that the wrinkling number $n$ increases from 5 to 7 as the shear load goes on. Meanwhile, the amplitude and wavelength keep on progressing. Comparing with the opening researches, the wrinkling numbers in the graphene with simply supported edge boundary are more than the one with free boundary. As $d$ increases, $\overline{\eta_{\alpha}}$ has a reduction and the maximum of outof-plane displacements $w_{E}$ grows. But at the eve fracture of graphene $(d=1.24 \mathrm{~nm}), w_{E}$ reaches $0.367 \mathrm{~nm}$. The variations of the wrinkling parameters over $d$ are shown in Figure 5. It is observed that two turning points occur on the curve owing to the generation of new wrinkles, respectively, which has influence on the mean value of wrinkling parameters. 


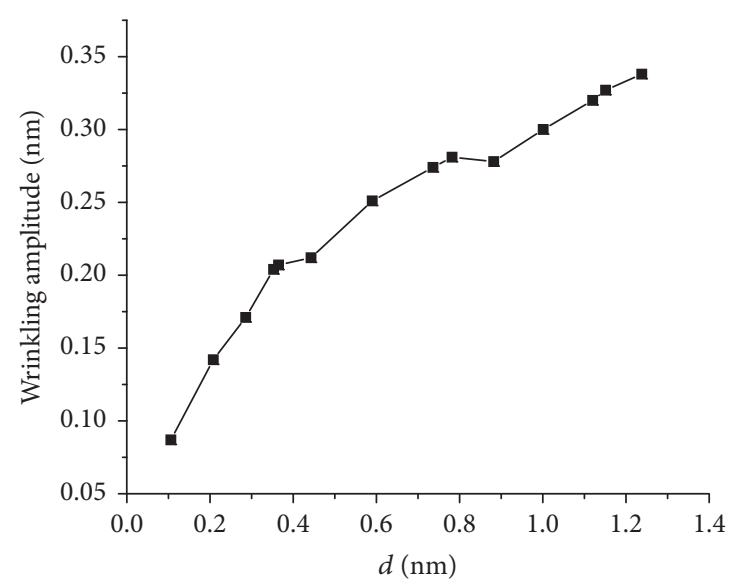

(a) Amplitude

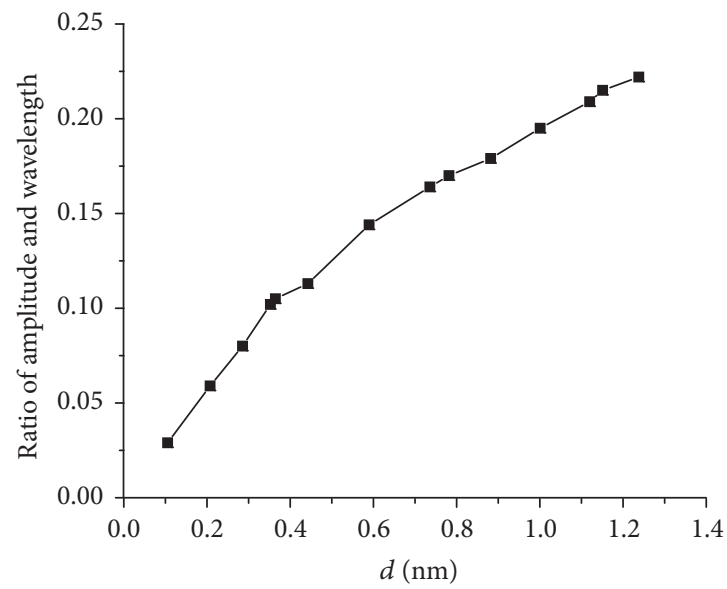

(c) Wrinkling ratio

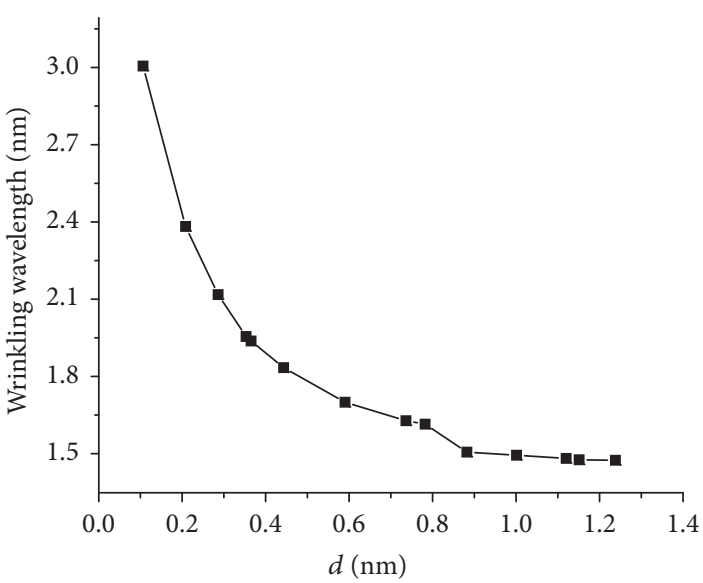

(b) Wavelength

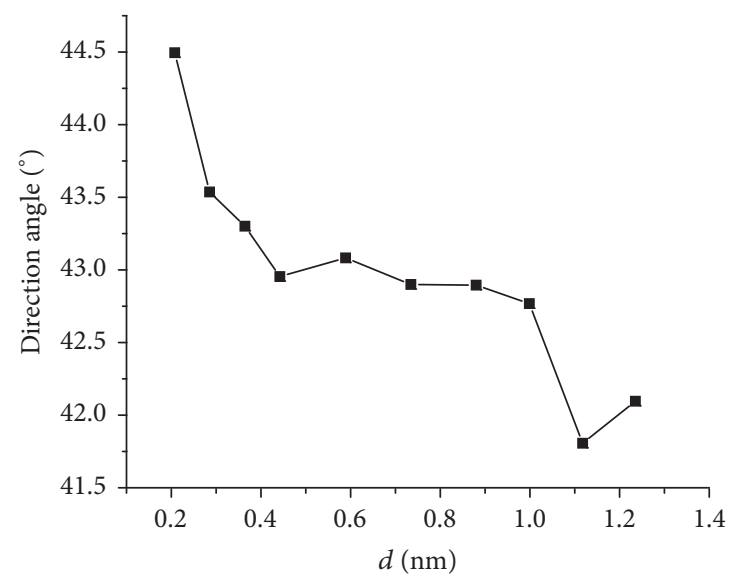

(d) Direction angle

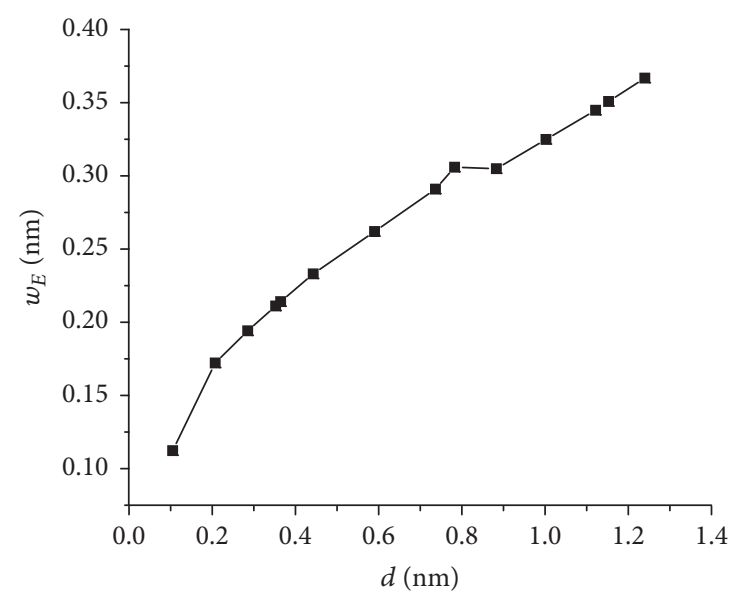

(e) $w_{E}$

Figure 5: Curves of wrinkling parameters over $d$.

TABLE 1: Wrinkling parameters over $d$.

\begin{tabular}{lccccc}
\hline$d(\mathrm{~nm})$ & $\overline{\eta_{A}}(\mathrm{~nm})$ & $\overline{\eta_{\lambda}}(\mathrm{nm})$ & $\overline{\eta_{A} / \eta_{\lambda}}$ & $n$ (crest, trough) & $\overline{\eta_{\alpha}}\left({ }^{\circ}\right)$ \\
\hline 0.21 & 0.142 & 2.409 & 0.059 & $5(3,2)$ & 44.493 \\
0.44 & 0.212 & 1.874 & 0.113 & $6(3,3)$ & 42.954 \\
0.88 & 0.278 & 1.554 & 0.179 & $7(4,3)$ & 0.172 \\
1.24 & 0.338 & 1.523 & 0.222 & $7(4,3)$ & 42.895 \\
\hline
\end{tabular}


TABLE 2: Wrinkling parameters in different aspect ratio $(d=0.88 \mathrm{~nm})$.

\begin{tabular}{lccccr}
\hline Aspect ratio & $\overline{\eta_{A}}(\mathrm{~nm})$ & $\overline{\eta_{\lambda}}(\mathrm{nm})$ & $\overline{\eta_{A} / \eta_{\lambda}}$ & $n$ (crest, trough) & $n \overline{\eta_{\lambda}} / L$ \\
\hline 1 & 0.321 & 1.543 & 0.208 & $2(1,1)$ & 0.762 \\
2 & 0.305 & 1.576 & 0.193 & $4(2,2)$ & 0.328 \\
3 & 0.283 & 1.570 & 0.181 & $7(4,3)$ & 0.3163 \\
5 & 0.292 & 1.644 & 0.178 & $11(5,6)$ & 0.904 \\
8 & 0.286 & 1.661 & 0.172 & $18(9,9)$ & 0.893 \\
13 & 0.278 & 1.676 & 0.166 & $30(15,15)$ & 0.922 \\
\hline
\end{tabular}

It shows that $\overline{\eta_{A}}, \overline{\eta_{A} / \eta_{\lambda}}$, and $w_{E}$ have increasing trend over $d$, while their increasing rates slow down at larger $d$. The wrinkling wavelength $\overline{\eta_{\lambda}}$ decreases rapidly at lower $d$ and then slowly reduces as $d$ increases. Due to the generation of the new wrinkle, the wrinkling angle $\overline{\eta_{\alpha}}$ exhibits a slight increment.

The graphene initially undergoes in-plane deformation once subjected to shear loading. As the loading displacement increases, owing to the finite thickness of graphene, the graphene displays out-of-plane deformation to release the strain energy and then forms the wrinkles to reach a lowenergy configuration. Based on the elasticity theory [23], the strain energy per unit area of wrinkled graphene can be represented by

$$
\bar{U}=\overline{U_{s}}+\overline{U_{b}},
$$

where $\overline{U_{s}}$ and $\overline{U_{b}}$ are stretching and bending terms of the energy, respectively. They can be expressed as

$$
\begin{aligned}
& \overline{U_{b}}=\frac{1}{2} \frac{E t^{3}}{12\left(1-v^{2}\right)} \frac{\pi^{4} A^{2}}{4 \lambda^{4}}, \\
& \overline{U_{s}}=\frac{E t}{2} \frac{\gamma^{2}}{4}+\frac{E t}{2} \frac{\pi^{2} A^{2} \gamma}{16 H^{2}},
\end{aligned}
$$

where $\gamma=d / H$ and $H$ and $t$ are the width and thickness of graphene. $A$ and $\lambda$ represent the wrinkling amplitude and wavelength. $E$ and $v$ are Young's modulus and Poisson's ratio of graphene.

Use the relation of

$$
A=\frac{\sqrt{2(1-v)}}{\pi} \lambda .
$$

The strain energy per unit area $\bar{U}$ can be expressed in terms of the wrinkling amplitude $A$ as

$$
\bar{U}=\frac{E t \gamma}{2}\left(\frac{(1-\nu) \gamma t^{2}}{12(1+\nu) A^{2}}+\frac{\pi^{2} A^{2}}{16 H^{2}}\right) .
$$

It is known, from (6), the energy increases as the wrinkling amplitude rises. After a new wrinkle is produced, a reduction of the wrinkling amplitude occurs, as seen in Figure 5(a). The reduction of wrinkling amplitude means that the energy of the new wrinkling configuration is lower than the ones before the new wrinkle occurs, which implies that a more stable wrinkling configuration is formed. As the loading continues, the energy keeps elevating and pushing the progress of the wrinkling. Until the occurrence of second new wrinkle, the energy decreases and forms a stable configuration. It can be observed that the formation of wrinkling is a low-energy process.

3.1. Aspect Ratio Effects. We define the aspect ratio as the ratio of length to width of the graphene sheet with a fixed width of $4.025 \mathrm{~nm}$. The effect on graphene wrinkling of chosen aspect ratio in ranges of 1 to 13 is studied in this paper. The wrinkling parameters, patterns, and atoms displacements in cross-line under same $d=0.88 \mathrm{~nm}$ are shown in Table 2 and Figures 6 and 7. The parameter $n \overline{\eta_{\lambda}} / L$ stands for the extent of wrinkling in graphene, where $L$ represents the length of graphene sheet. It is observed that the wrinkling amplitude $\overline{\eta_{A}}$ and ratio $\overline{\eta_{A} / \eta_{\lambda}}$ decrease with the increasing aspect ratio. On the contrary, the wrinkling wavelength $\overline{\eta_{\lambda}}$, wrinkling number $n$, and extent parameter $n \overline{\eta_{\lambda}} / L$ increase as the aspect ratio increases. The wrinkles become stubby and flat with a wider coverage extent, as shown in Figures 6 and 7.

3.2. Temperature Effects. We investigate the temperature effects on wrinkling parameters and patterns of rectangular graphene with aspect ratio of 3 under temperature ranging from $0.01 \mathrm{~K}$ to $600 \mathrm{~K}$. The wrinkling parameters and patterns at $d=0.47 \mathrm{~nm}$ are listed in Table 3 and Figure 8. As seen in Table 3, it shows that temperature has great influence on graphene wrinkling process and wrinkling parameters. The existence of temperature intensifies the thermal vibration of graphene atoms, which significantly influences the wrinkling deformation process. As a consequence, severe variation of the wrinkling parameters occurs as the temperature rises. Generally, it displays an increasing trend of $\overline{\eta_{A}}$ and $\overline{\eta_{A} / \eta_{\lambda}}$ as temperature increases. $\overline{\eta_{\lambda}}$ and $n \overline{\eta_{\lambda}} / L$ are enlarged with the increasing temperature, when temperature is less than $400 \mathrm{~K}$, after which $\overline{\eta_{\lambda}}$ and $n \overline{\eta_{\lambda}} / L$ reduce as temperature increases. Obvious discrepancy between wrinkling patterns under different temperatures is observed from Figure 8, indicating the significant temperature effect on wrinkling pattern, which affects the wrinkling parameters.

We perform an annealing treatment simulation to wrinkling graphene and investigate the effect of annealing on graphene wrinkling. As seen in Figure 9, after graphene wrinkles, the temperature decreases to $0.01 \mathrm{~K}$ gradually. After the temperature is maintained in $0.01 \mathrm{~K}$, the wrinkling parameters and configuration are obtained and shown in Table 3 and Figure 8. The wrinkling parameters under different temperature situation become close after annealing 


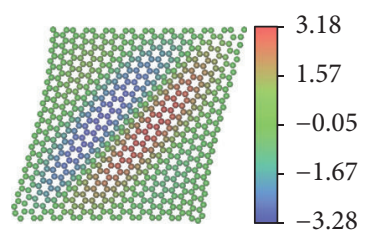

(a)

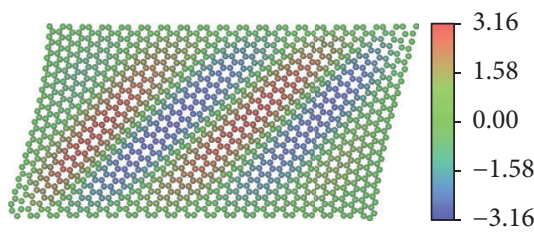

(b)

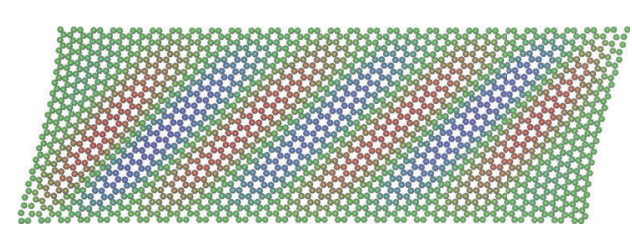

(c)

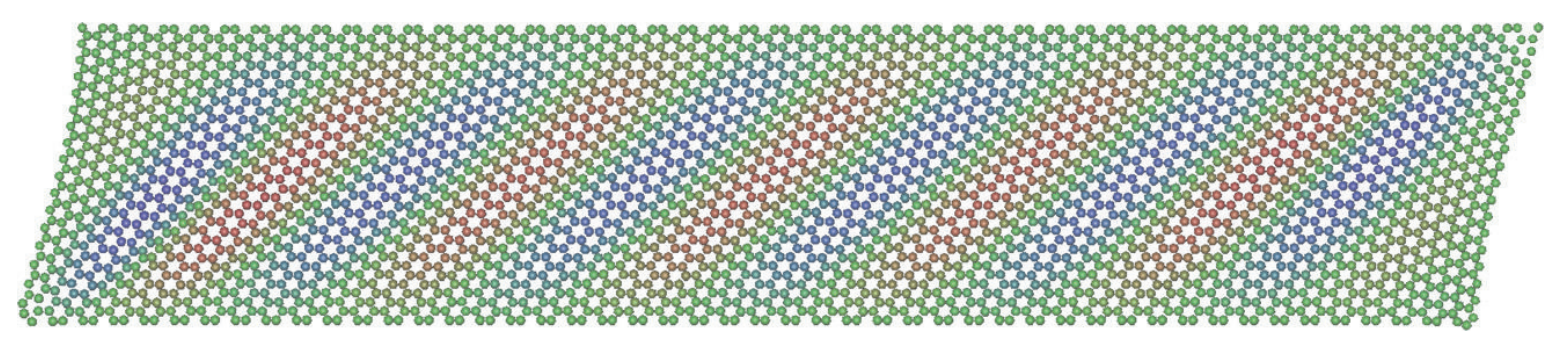

(d)

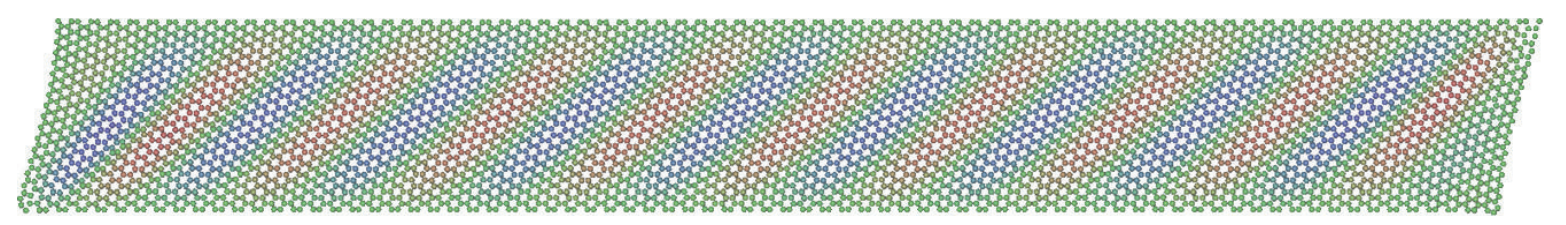

(e)
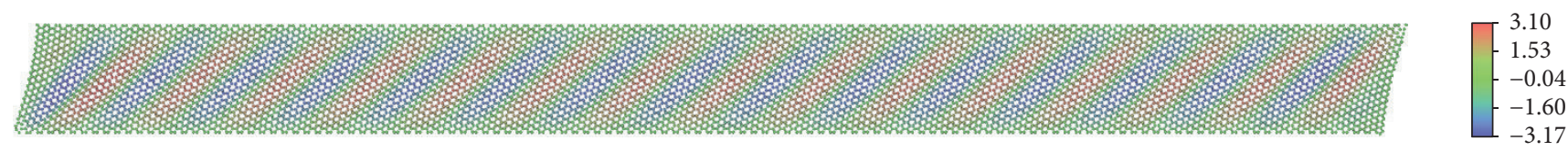

(f)

Figure 6: Wrinkling patterns in different aspect ratio $(d=0.88 \mathrm{~nm})$, unit: A. (a) Aspect ratio 1. (b) Aspect ratio 2. (c) Aspect ratio 3. (d) Aspect ratio 5. (e) Aspect ratio 8. (f) Aspect ratio 13.

TABLE 3: Wrinkling parameters under different temperatures $(d=0.47 \mathrm{~nm})$.

\begin{tabular}{|c|c|c|c|c|c|c|}
\hline Temperature $(\mathrm{K})$ & $\overline{\eta_{A}}(\mathrm{~nm})$ & $\overline{\eta_{\lambda}}(\mathrm{nm})$ & $\overline{\eta_{A} / \eta_{\lambda}}$ & $n$ (crest, trough) & $n \overline{\eta_{\lambda}} / L$ & $w_{E}$ \\
\hline 0.01 & 0.219 & 1.859 & 0.118 & $6(3,3)$ & 0.918 & 0.276 \\
\hline 100 & 0.226 & 1.823 & 0.124 & $6(3,3)$ & 0.900 & 0.242 \\
\hline 100 to 0.01 & 0.217 & 1.869 & 0.116 & $6(3,3)$ & 0.930 & 0.237 \\
\hline 200 & 0.218 & 1.836 & 0.119 & $6(3,3)$ & 0.906 & 0.250 \\
\hline 200 to 0.01 & 0.217 & 1.867 & 0.117 & $6(3,3)$ & 0.929 & 0.237 \\
\hline 300 & 0.234 & 1.866 & 0.126 & $6(3,3)$ & 0.921 & 0.275 \\
\hline 300 to 0.01 & 0.218 & 1.875 & 0.116 & $6(3,3)$ & 0.934 & 0.237 \\
\hline 400 & 0.237 & 1.918 & 0.124 & $6(3,3)$ & 0.947 & 0.271 \\
\hline 400 to 0.01 & 0.217 & 1.871 & 0.116 & $6(3,3)$ & 0.931 & 0.237 \\
\hline 500 & 0.237 & 1.881 & 0.126 & $6(3,3)$ & 0.928 & 0.281 \\
\hline 500 to 0.01 & 0.217 & 1.871 & 0.116 & $6(3,3)$ & 0.931 & 0.237 \\
\hline 600 & 0.236 & 1.860 & 0.126 & $6(3,3)$ & 0.918 & 0.313 \\
\hline 600 to 0.01 & 0.217 & 1.868 & 0.117 & $6(3,3)$ & 0.930 & 0.237 \\
\hline
\end{tabular}

treatment, indicating that the annealing treatment eliminates the effect of temperature on wrinkling parameters. But, as for wrinkling configuration, the wrinkling pattern is still the same as the one before annealing treatment. It means that the annealing treatment cannot recover the wrinkling pattern to low temperature.
Based on the elasticity theory, the graphene structure wrinkles when it is subjected to critical strain. With the existence of temperature, the atoms of graphene exhibit thermal motion, which produces nonnegligible atomic out-ofplane displacement compared to the thickness of graphene. Therefore ripples are formed upon the graphene [6]. The 

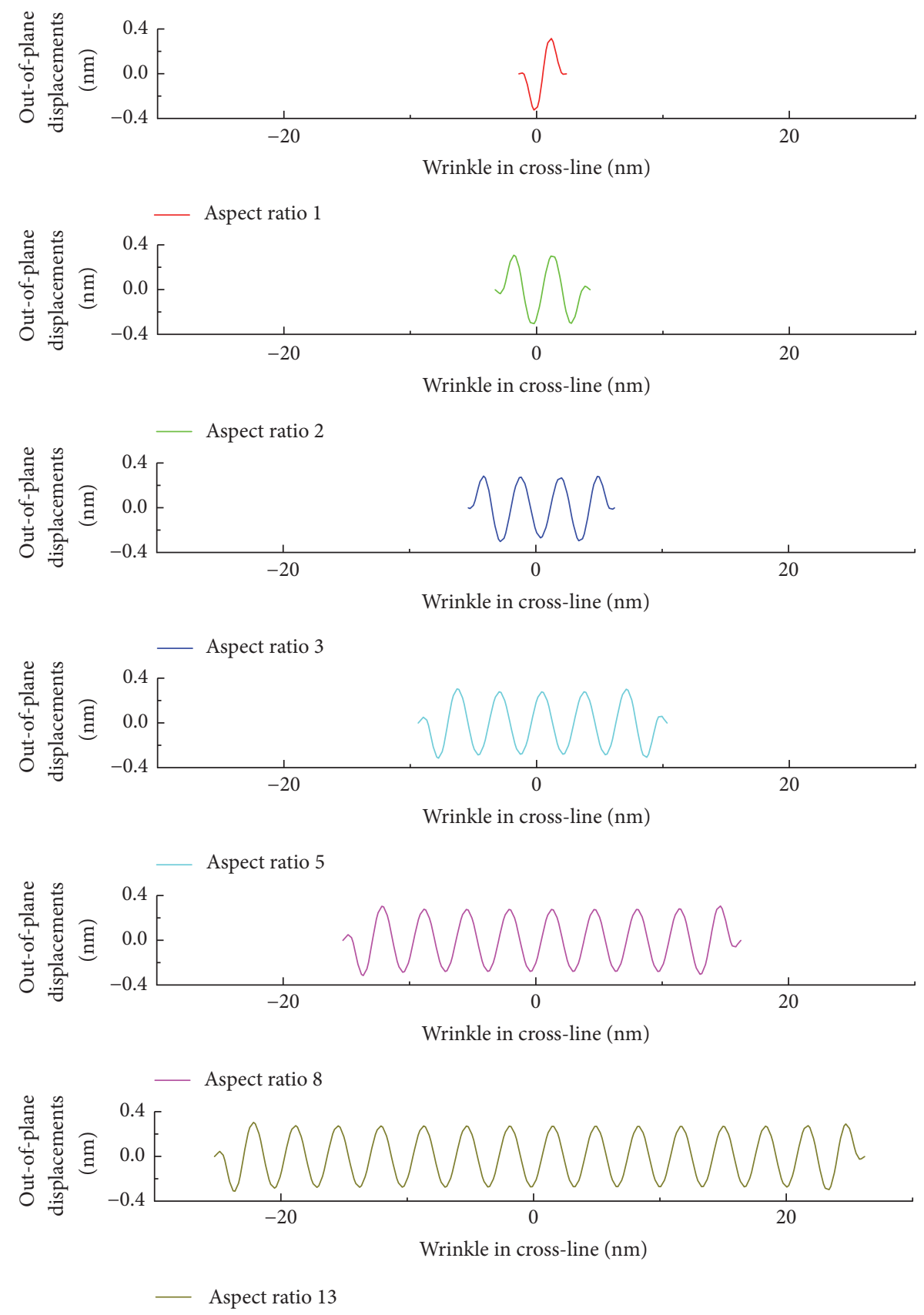

Figure 7: Atoms displacements in cross-line I with different aspect ratio $(d=0.88 \mathrm{~nm})$.

out-of-plane of the wrinkling bifurcation point significantly influences the wrinkling configuration.

3.3. Loading Velocity Effects. The wrinkling of graphene sheet with aspect ratio of 3 under different loading velocity is investigated in this section. The loading velocity ranges from $6 \times 10^{-5}$ to $2.2 \times 10^{-4} \mathrm{~nm} / \mathrm{ps}$ and the other simulation settings remain unchanged. The wrinkling parameters and patterns of graphene wrinkles under $d=0.88 \mathrm{~nm}$ are shown in Table 4 and Figure 10. It shows that as the loading velocity increases the wrinkling parameters change slightly, indicating that wrinkling parameters have low dependency upon the loading velocity. Based on the results, in all loading velocity cases graphene generates the same number wrinkles, but the wrinkling patterns are different as seen in Table 4 and Figure 10. It illustrates that loading velocity has impact on wrinkling patterns but slightly affects wrinkling parameters. The loading velocity not only affects the mechanical property performance of materials but also affects the deformation response of the materials [24, 25]. Under different loading velocity, the relaxation time for the rearrangement of carbon atoms and bonds is different. On the eve of the wrinkling of 


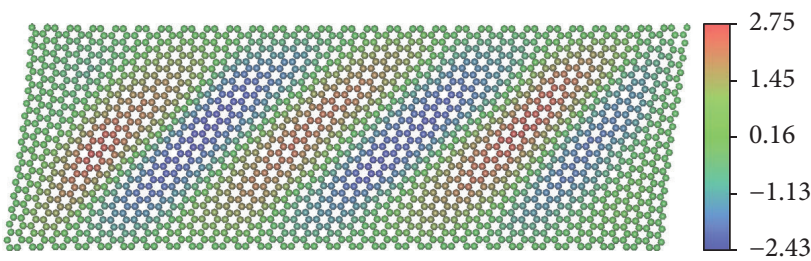

(a) $300 \mathrm{~K}$

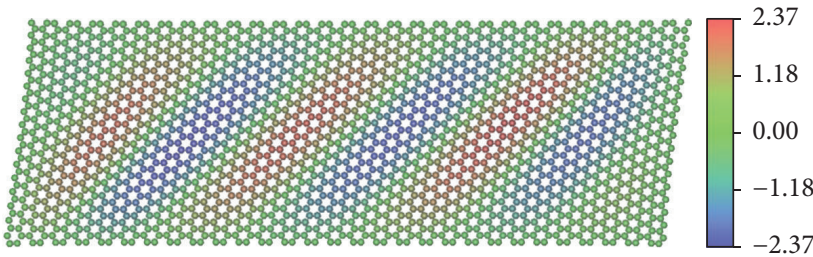

(c) $300 \mathrm{~K}$ anneals to $0.01 \mathrm{~K}$

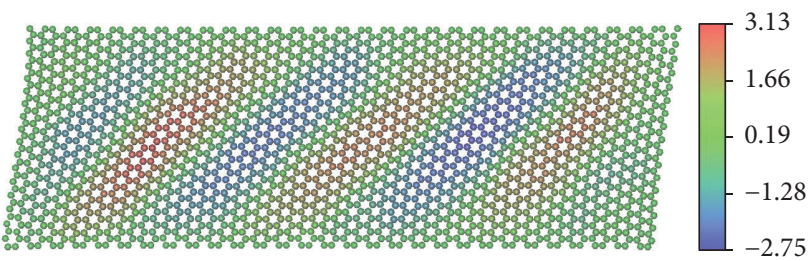

(b) $600 \mathrm{~K}$

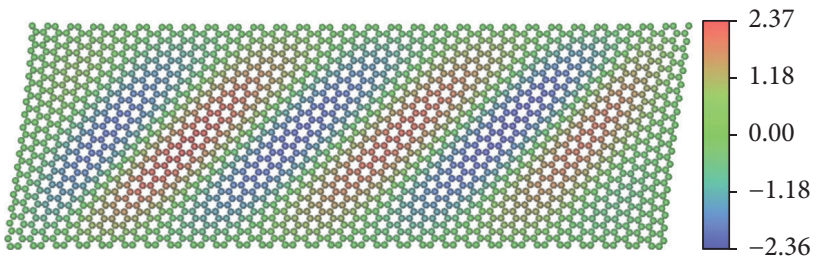

(d) $600 \mathrm{~K}$ anneals to $0.01 \mathrm{~K}$

FIGURE 8: Wrinkling patterns under different temperatures $(d=0.47 \mathrm{~nm})$, unit: $\AA$.

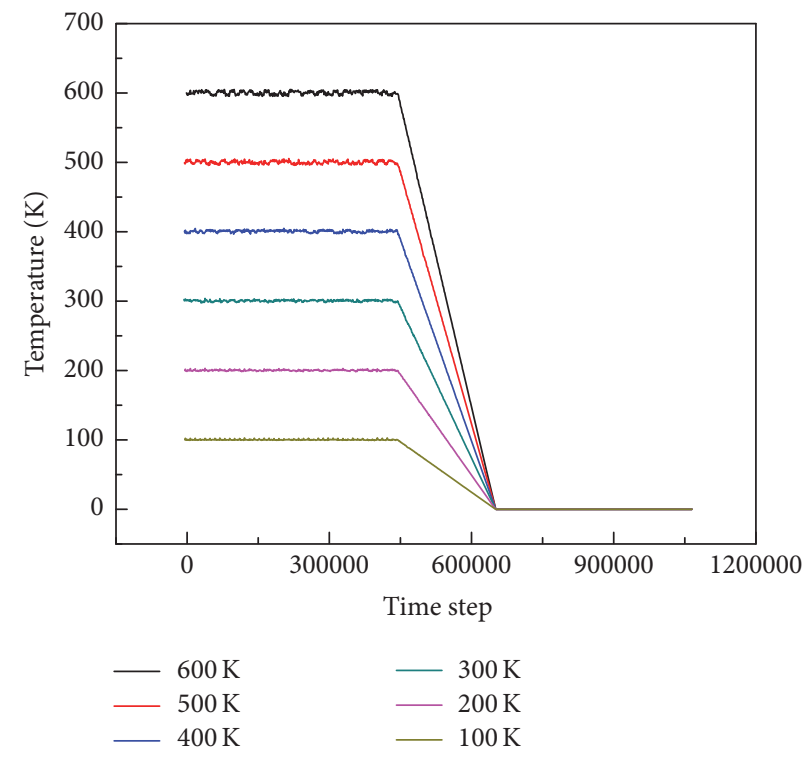

Figure 9: Annealing treatment simulation, the system temperature decreases to $0.01 \mathrm{~K}$ gradually.

TABLE 4: Wrinkling parameters under different loading velocity $(d=0.88 \mathrm{~nm})$.

\begin{tabular}{lcccrr}
\hline $\begin{array}{l}\text { Velocity } \\
\left(10^{-5} \mathrm{~nm} / \mathrm{ps}\right)\end{array}$ & $\overline{\eta_{A}}(\mathrm{~nm})$ & $\overline{\eta_{\lambda}}(\mathrm{nm})$ & $\overline{\eta_{A} / \eta_{\lambda}}$ & $n$ (crest, trough) & $n \overline{\eta_{\lambda}} / L$ \\
\hline 6 & 0.280 & 1.580 & 0.178 & $7(3,4)$ & 0.916 \\
8 & 0.281 & 1.573 & 0.179 & $7(3,4)$ & 0.912 \\
10 & 0.283 & 1.570 & 0.181 & $7(4,3)$ & 0.306 \\
12 & 0.279 & 1.582 & 0.176 & $7(4,3)$ & 0.910 \\
14 & 0.280 & 1.575 & 0.178 & $7(3,4)$ & 0.917 \\
18 & 0.280 & 1.576 & 0.178 & $7(4,3)$ & 0.913 \\
22 & 0.280 & 1.569 & 0.179 & $7(3,4)$ & 0.914 \\
\hline
\end{tabular}




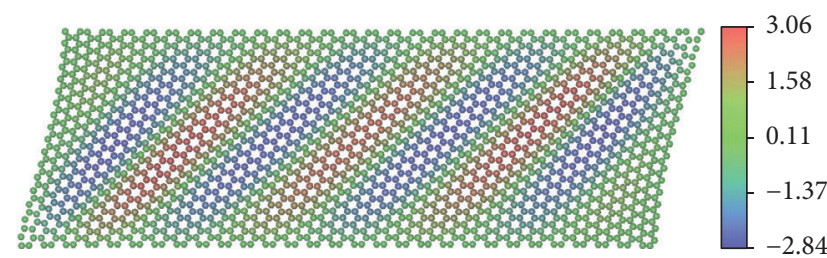

(a)

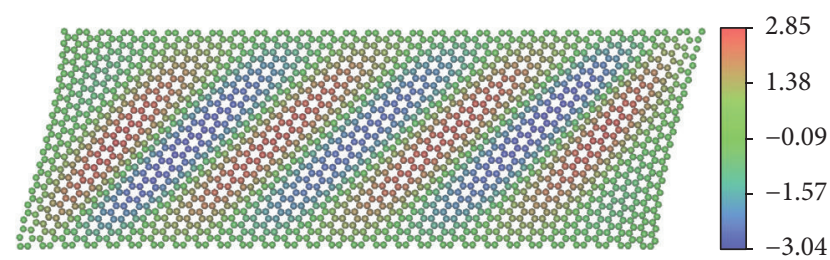

(b)

FIGURE 10: Wrinkling patterns under different loading velocity $(d=0.88 \mathrm{~nm})$, unit: $\AA$. (a) $8 \times 10^{-5} \mathrm{~nm} / \mathrm{ps}$. (b) $1.8 \times 10^{-4} \mathrm{~nm} / \mathrm{ps}$.

graphene, the rearrangement situation of the carbon atoms has great influence on the wrinkling configuration after graphene wrinkles.

\section{Conclusions}

In summary, in the present paper the wrinkling of shearloaded rectangular SLGS with simply supported boundary condition is investigated through MD simulations. It reveals that, due to the graphene shape and edge boundary condition, the wrinkles are produced on the graphene surface, and the new wrinkles are generated from the right edge driving the wrinkles to propagate to the left. Generation of new wrinkles changes the direction angles of the wrinkles group. The wrinkling revolution process is divided into five stages: (I) prophase stage, (II) prometaphase stage, (III) metaphase stage, (IV) anaphase stage, and (V) telophase stage. The wrinkling amplitude, maximum of out-of-plane displacements, and ratio of amplitude and wavelength rise with the increasing shear displacements, but the wrinkling wavelength reduces in general. The wrinkling formation is the energyreleasing process during the deformation of graphene under shear loading. The aspect ratio of graphene and temperature greatly affects the graphene wrinkling. The larger aspect ratio makes the wrinkles flatter and wider extent coverage. Wrinkling deformation of graphene is significantly influenced by temperature due to the intensive atomic thermal vibration. And it is found that the annealing process could eliminate the temperature effect on wrinkling parameter but could not recover graphene wrinkling configuration to low temperature. The loading velocity has little effect on wrinkling parameters but affects the wrinkling patterns. Because of the rearrangement of carbon atoms, the loading velocity has influence on the wrinkling configuration when graphene wrinkles.

\section{Competing Interests}

The authors declare that they have no competing interests.

\section{Acknowledgments}

The authors thank the support from the National Natural Science Foundation of China (11272123 and 11472108).

\section{References}

[1] A. K. Geim and K. S. Novoselov, "The rise of graphene," Nature Materials, vol. 6, no. 3, pp. 183-191, 2007.

[2] L. Zhang, Y. Shao, Z. Tu et al., "Graphene sensing an inhomogeneous strain due to the surface relief in FeNiCoTi shape memory alloy," Journal of Raman Spectroscopy, vol. 45, no. 1, pp. 1-6, 2014.

[3] K. Kim, J.-Y. Choi, T. Kim, S.-H. Cho, and H.-J. Chung, "A role for graphene in silicon-based semiconductor devices," Nature, vol. 479, no. 7373, pp. 338-344, 2011.

[4] T. Zhang, Q. Xue, S. Zhang, and M. Dong, "Theoretical approaches to graphene and graphene-based materials," Nano Today, vol. 7, no. 3, pp. 180-200, 2012.

[5] C. Liu, F. Li, M. Lai-Peng, and H.-M. Cheng, "Advanced materials for energy storage," Advanced Materials, vol. 22, no. 8, pp. E28-E62, 2010.

[6] J. C. Meyer, A. K. Geim, M. I. Katsnelson, K. S. Novoselov, T. J. Booth, and S. Roth, "The structure of suspended graphene sheets," Nature, vol. 446, no. 7131, pp. 60-63, 2007.

[7] T. M. Paronyan, E. M. Pigos, G. Chen, and A. R. Harutyunyan, "Formation of ripples in graphene as a result of interfacial instabilities," ACS Nano, vol. 5, no. 12, pp. 9619-9627, 2011.

[8] N. Liu, Z. Pan, L. Fu, C. Zhang, B. Dai, and Z. Liu, "The origin of wrinkles on transferred graphene," Nano Research, vol. 4, no. 10, pp. 996-1004, 2011.

[9] Z. Pan, N. Liu, L. Fu, and Z. Liu, "Wrinkle engineering: a new approach to massive graphene nanoribbon arrays," Journal of the American Chemical Society, vol. 133, no. 44, pp. 17578-17581, 2011.

[10] H. Vandeparre, M. Piñeirua, F. Brau et al., "Wrinkling hierarchy in constrained thin sheets from suspended graphene to curtains," Physical Review Letters, vol. 106, no. 22, Article ID 224301, 2011.

[11] J. A. Baimova, S. V. Dmitriev, K. Zhou, and A. V. Savin, "Unidirectional ripples in strained graphene nanoribbons with clamped edges at zero and finite temperatures," Physical Review B-Condensed Matter and Materials Physics, vol. 86, no. 3, Article ID 035427, 2012.

[12] L. Meng, Y. Su, D. Geng et al., "Hierarchy of graphene wrinkles induced by thermal strain engineering," Applied Physics Letters, vol. 103, no. 25, Article ID 251610, 2013.

[13] K. Chu, W.-S. Li, and H. Dong, "Role of graphene waviness on the thermal conductivity of graphene composites," Applied Physics A: Materials Science and Processing, vol. 111, no. 1, pp. 221-225, 2013.

[14] M. Ahmad, H. An, Y. S. Kim et al., "Nanoscale investigation of charge transport at the grain boundaries and wrinkles in 
graphene film," Nanotechnology, vol. 23, no. 28, Article ID 285705, 2012.

[15] Y. Guo and W. Guo, "Electronic and field emission properties of wrinkled graphene," Journal of Physical Chemistry C, vol. 117, no. 1, pp. 692-696, 2013.

[16] Z. Ye, C. Tang, Y. Dong, and A. Martini, "Role of wrinkle height in friction variation with number of graphene layers," Journal of Applied Physics, vol. 112, no. 11, Article ID 116102, 2012.

[17] Y. Dong, S. Li, H. Xu et al., "Wrinkled-graphene enriched $\mathrm{MoO}_{3}$ nanobelts with increased conductivity and reduced stress for enhanced electrochemical performance," Physical Chemistry Chemical Physics, vol. 15, no. 40, pp. 17165-17170, 2013.

[18] H. Qin, Y. Sun, J. Z. Liu, and Y. Liu, "Mechanical properties of wrinkled graphene generated by topological defects," Carbon, vol. 108, pp. 204-214, 2016.

[19] L. Ortolani, E. Cadelano, G. P. Veronese et al., "Folded graphene membranes: mapping curvature at the nanoscale," Nano Letters, vol. 12, no. 10, pp. 5207-5212, 2012.

[20] V. E. Calado, G. F. Schneider, A. M. M. G. Theulings, C. Dekker, and L. M. K. Vandersypen, "Formation and control of wrinkles in graphene by the wedging transfer method," Applied Physics Letters, vol. 101, no. 10, 2012.

[21] M. Lanza, Y. Wang, A. Bayerl et al., “Tuning graphene morphology by substrate towards wrinkle-free devices: experiment and simulation," Journal of Applied Physics, vol. 113, no. 10, Article ID 104301, 2013.

[22] D. W. Brenner, O. A. Shenderova, J. A. Harrison, S. J. Stuart, B. $\mathrm{Ni}$, and S. B. Sinnott, "A second-generation reactive empirical bond order (REBO) potential energy expression for hydrocarbons," Journal of Physics Condensed Matter, vol. 14, no. 4, pp. 783-802, 2002.

[23] W. Wong and S. Pellegrino, "Wrinkled membranes II: analytical models," Journal of Mechanics of Materials and Structures, vol. 1, no. 1, pp. 27-61, 2006.

[24] Z. Yang, Y. Huang, F. Ma, Y. Sun, K. Xu, and P. K. Chu, “Temperature and strain-rate effects on the deformation behaviors of nano-crystalline graphene sheets," European Physical Journal B, vol. 88, no. 5, article 135, 2015.

[25] M. Q. Chen, S. S. Quek, Z. D. Sha, C. H. Chiu, Q. X. Pei, and Y. W. Zhang, "Efects of grain size, temperature and strain rate on the mechanical properties of polycrystalline graphene-a molecular dynamics study," Carbon, vol. 85, pp. 135-146, 2015. 

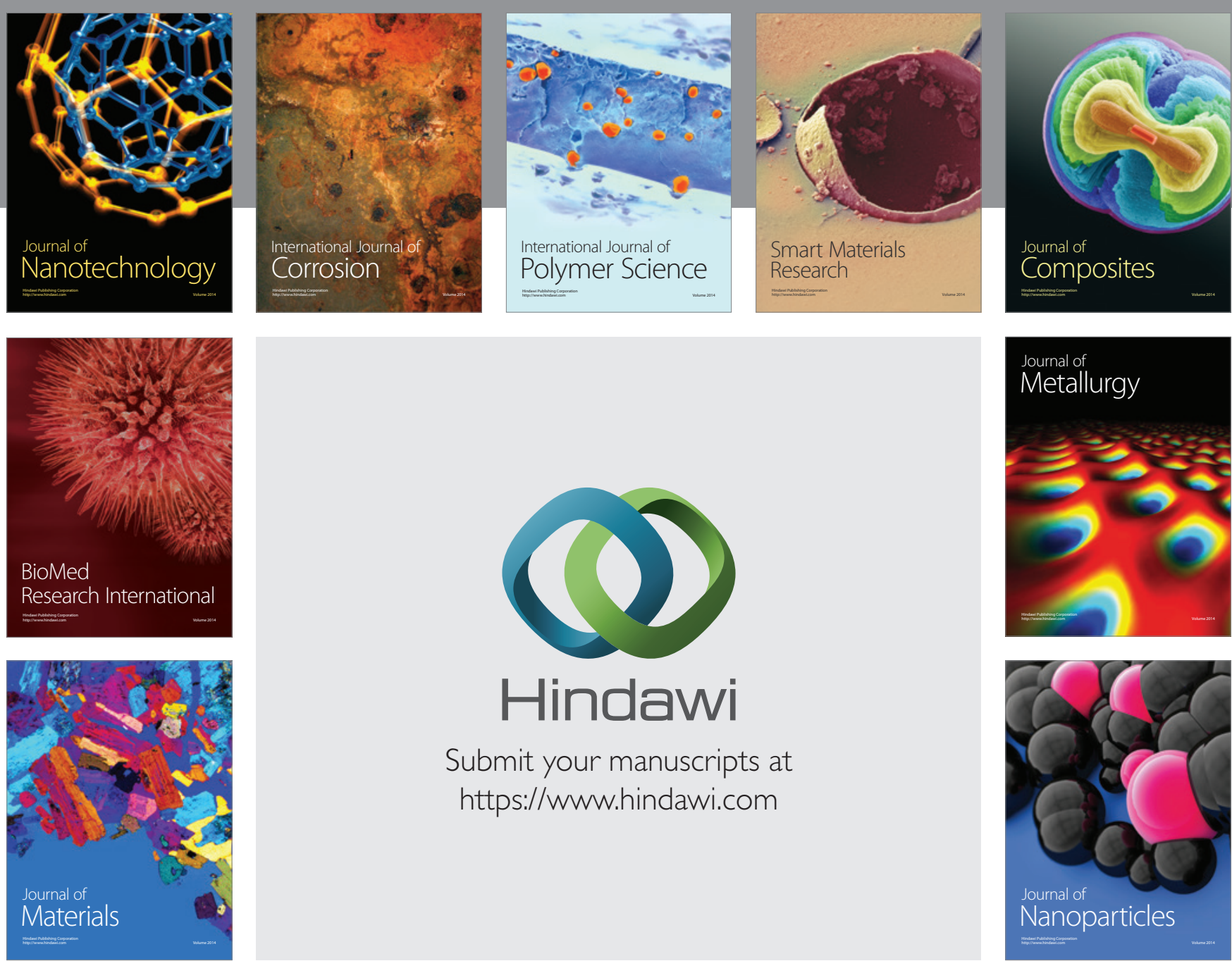

\section{Hindawi}

Submit your manuscripts at

https://www.hindawi.com

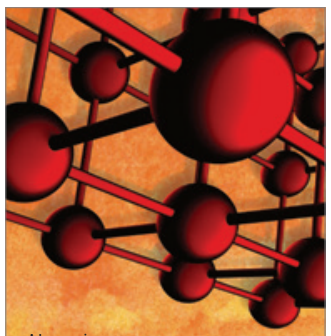

Materials Science and Engineering
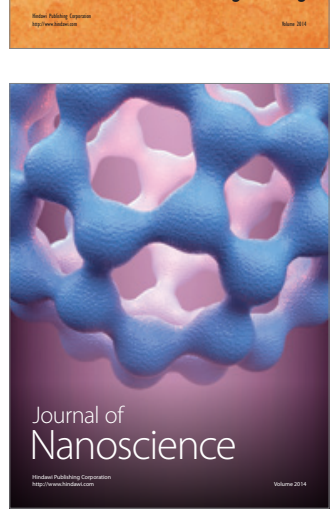
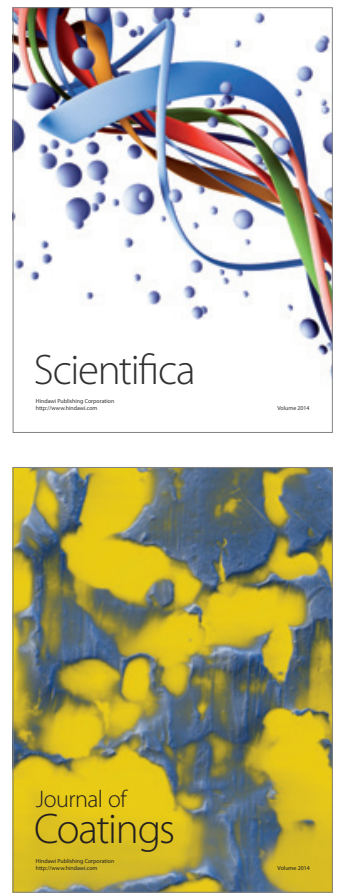
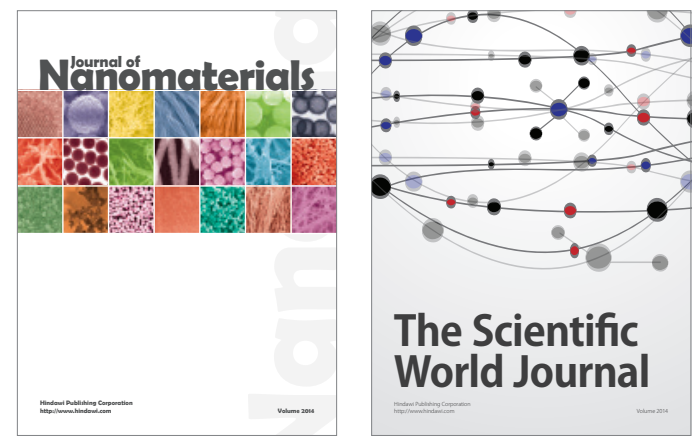

The Scientific World Journal
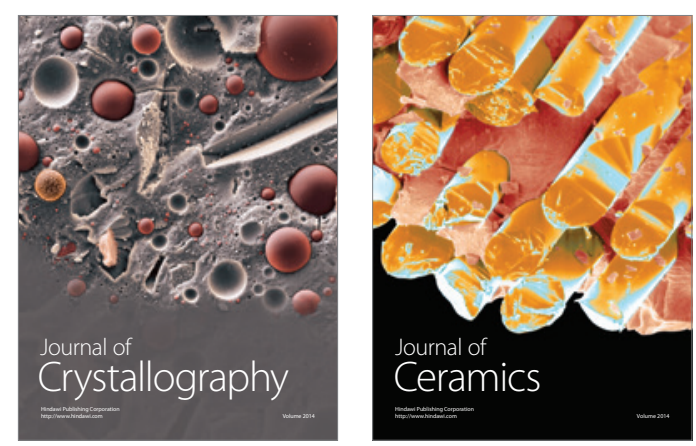
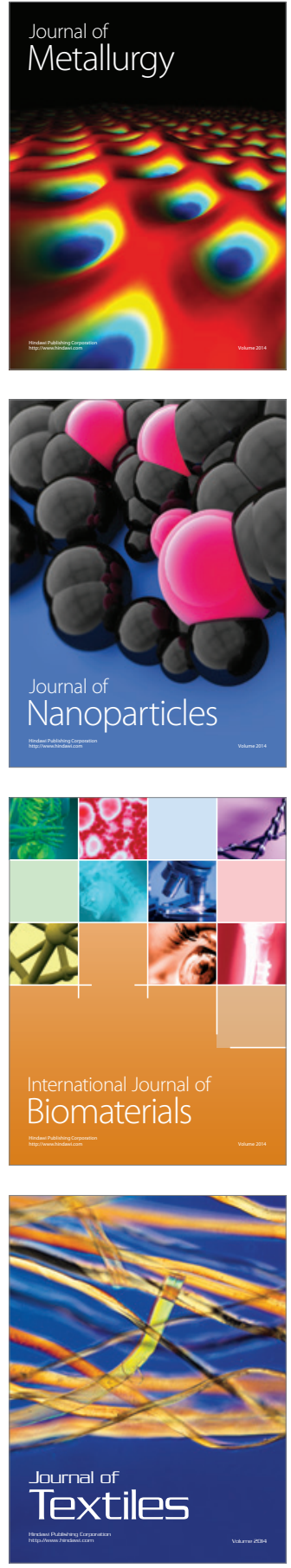